\title{
A NOTE ON PRIMITIVE RECURSIVE FUNCTIONS
}

\author{
JULIA ROBINSON
}

The purpose of this note is to prove three theorems on primitive recursive functions of one variable which are analogous to three earlier theorems on general recursive functions. Theorems 1, 2, and 3 parallel closely Theorems 4,5 , and 6 of GRF $\$ 4 .{ }^{1}$ We shall use the same notation as before, calling special attention to the fact that $J$, $K$, and $L$ are the Cantor pairing functions.

THEOREM 1. All primitive recursive functions of one variable can be obtained by starting with a certain two primitive recursive functions and repeatedly using the formulas

$$
F x=B A x, \quad F x=B^{x} 0
$$

to construct a new function from known functions $A$ and $B$.

Proof. As before, we need only show that a finite set of primitive recursive functions is sufficient, since these can then be reduced to two functions by pairing (GRF p. 712). Again, to every function $F$, we let correspond a function $F^{*}=J(F K, L)$. We shall show that if $F$ is defined by any of the three formulas

$$
F x=A x+B x, \quad F x=B A x, \quad F x=B^{x} 0,
$$

then $F^{*}$ can be obtained from $A^{*}, B^{*}$, and several specific functions by substitution and recursion alone. Now, by a theorem of R. M. Robinson, ${ }^{2}$ all primitive recursive functions of one variable can be obtained from $S$ and $K$ by successive additions, substitutions, and recursions of the form $F x=B^{x} 0$. Hence if $F$ is a primitive recursive function, then $F^{*}$ can be obtained from a suitable set of initial functions (including $S^{*}$ and $K^{*}$ ) by substitution and recursion. We also have $F=K F^{*} J(I, I)$. Therefore, if $K$ and $J(I, I)$ are included in the initial set of functions, we can obtain not only all the star functions corresponding to primitive recursive functions but also all the primitive functions themselves.

We still need to show how $F^{*}$ can be obtained from $A^{*}$ and $B^{*}$. Referring to GRF, p. 713 , we see that $(B A)^{*}=B^{*} A^{*}$ and $(A+B)^{*}$

Received by the editors November 12, 1954.

${ }^{1}$ Julia Robinson, General recursive functions, Proc. Amer. Math. Soc. vol. 1 (1950) pp. 703-718.

2 R. M. Robinson, Primitive recursive functions. II, Proc. Amer. Math. Soc. vol. 6 (1955) pp. 663-666. 
$=G_{3} B^{*} G_{2} A^{*} G_{1}$ where $G_{1}=J(K, I), G_{2}=J(K L, I)$, and $G_{3}=J(K+K L$, $L L L)$. Let $F$ be defined by $F x=B^{x} 0$. Then $F^{*} x=J\left(B^{K x} 0, L x\right) . F^{*}$ cannot in general be defined by recursion, since $F^{*}$ is unbounded but is not necessarily univalent. We turn instead to a related function $J(F K, I)$ which is univalent and from which we can obtain $F^{*}$ by substitution, since $F^{*}=J(K, L L) J(F K, I)$. Now any univalent function which vanishes at zero can be expressed as $M^{x} 0$ for a properly chosen function $M$. We first determine $M$ so that $J(F K x, x)=M^{x} 0$. We shall then show that $M=H B^{*}$ where $H$ is a fixed function independent of $B$. We need to express $J(F K S x, S x)$ in terms of $J(F K x, x)$ $=J\left(B^{K x} 0, x\right)$, making use of the fact that $K S x$ is either $S K x$ or 0 . Hence,

$$
J(F K S x, S x)=J\left(\operatorname{sgn} K S x \cdot B B^{K x} 0, S x\right) .
$$

However, $S x=S L J(F K x, x)$ and $B^{K x} 0=K J(F K x, x)$, so $M u$ $=J(\operatorname{sgn} K S L u \cdot B K u, S L u)$. We still must show that $M=H B^{*}$ where $H$ is independent of $B$. This can easily be done, since $B K u=K B^{*} u$ and $L u=L B^{*} u$ and $M u=J\left(\operatorname{sgn} K S L B^{*} u \cdot K B^{*} u, S L B^{*} u\right)=H B^{*} u$ where $H=J(\operatorname{sgn} K S L \cdot K, S L){ }^{3}$ This completes the proof, for we have shown that any set of initial functions which is sufficient to yield $S^{*}, K^{*}, K, J(I, I), G_{1}, G_{2}, G_{3}, J(K, L L)$ and $H$ by substitution and recursion will generate all primitive recursive functions.

We now turn to the problem of replacing these nine functions by fewer functions of a simpler nature than the complicated function obtained from the nine by pairing.

THEOREM 2. All primitive recursive functions of one variable can be obtained by substitution and recursion of the form $F x=B^{x} 0$ from the four functions $J(L K, K L), J(I, S O), J(K+K L, I)$, and $J(\operatorname{sgn} K L \cdot K, L)$.

Proof. It is sufficient to show that we can define the nine functions listed at the end of the proof of Theorem 1. First of all,

$J(L K, K L) J(K+K L, I) J(I, S O)=J(L K, K L) J(I, J(I, S O))=J(L, I)$.

Hence by the lemma on page 714 of GRF, we can obtain all functions $J(M, N)$ where $M$ and $N$ are obtained from $I, K$, and $L$ by substitution. Since the Cantor pairing functions are one-to-one, we also obtain $K=J(K K, L K)$.

We still have three functions to define which we can obtain by substitution in the following way:

${ }^{3}$ Here, of course, we let $K L \cdot K$ denote the function $G$ defined by $G x=K L x \cdot K x$. 


$$
\begin{gathered}
J(K, L L L L) J(K+K L, I)=J(K+K L, L L L), \\
J(K, L L L) J(K+K L, I) J(L, K) J(I, S O) \\
=J(K+K L, L L) J(S O, I)=J(S K, L), \\
J(\operatorname{sgn} K L \cdot K, L) J(L, \quad K) J(S K, L) J(L, K) \\
=J(\operatorname{sgn} K L \cdot K, L) J(L, K) J(S L, K) \\
=J(\operatorname{sgn} K L \cdot K, L) J(K, S L)=J(\operatorname{sgn} K S L \cdot K, S L) .
\end{gathered}
$$

We shall now show that it is impossible to reduce the two initial functions of Theorem 1 to a single function of any sort, even if we allow the more general form of recursion, $F x=B^{x} a$.

Leмma. Consider the class of functions generated from a single function $A$ by repeated substitution and recursion of the form $F x=B^{x} a$, where various values of a may be used. If $F$ is any function of this class, then one of the following two conditions is satisfied:

(1) $F=A^{k}$ for some positive integer $k$.

(2) There exist a number $a$ and a finite exceptional set $\alpha$ such that for every $x$, either $F x=A^{n} a$ for some positive $n$ or $F x \in \alpha$.

Proof. To show this, we need only check that if $G$ and $H$ satisfy either condition, then $G H$ and $G^{x} b$ also satisfy one of the alternatives. First consider $G H$. Clearly, if $G$ and $H$ both satisfy (1), so also does $G H$. If $G$ satisfies (2), then $G H$ satisfies (2), since the range of $G H$ is included in the range of $G$. Finally, suppose $G$ satisfies (1) and $\mathrm{H}$ satisfies (2), i.e. there exist $k, a$, and a finite set $\alpha$, so that $G=A^{k}$ and for every $x$, either there is an $n$ such that $H x=A^{n} a$ or $H x \in \alpha$. It follows that for every $x, G H=A^{k+n} a$ or $G H x$ belongs to a finite set, namely the map of $\alpha$ by $G$. Hence $G H$ satisfies (2).

Now consider $F x=G^{x} b$. If $G$ satisfies (1), then $F x=A^{k x} b$, so $F$ satisfies (2). If $G$ satisfies (2), so also does $F$ since every value of $F$ is a value of $G$ except possibly for $F 0=b$.

TheOREM 3. There is no single function $A$ from which all primitive recursive functions can be obtained by substitution and recursion of the form $F x=B^{x} a$.

Proof. Case 1. $A$ assumes only a finite number of values more than once. We shall show that every function obtained from $A$ has this property. Functions obtained by recursion are either bounded or univalent and hence do have the stated property. Finally, if $G$ and $H$ both have this property, we must show that $G H$ does also. Clearly, if $G H x_{1}=G H x_{2}$, then either $H x_{1}=H x_{2}$ or if we put $y_{1}=H x_{1}$ and $y_{2}=H x_{2}$, we have $y_{1} \neq y_{2}$ and $G y_{1}=G y_{2}$. Hence the set of possible 
values $z$ for which $z=G H x$ has more than one solution must be finite. Thus in Case 1, a function such as $K$ cannot be defined and hence not all primitive recursive functions can be obtained from $A$.

Case 2. $A$ assumes infinitely many values more than once. In this case, we use the lemma. Notice that if $H$ is not univalent, then $G H$ is not univalent. Hence no power of $A$ is univalent. On the other hand, $A^{x} a$ omits infinitely many values. For if $A u=A v$, and $A^{x} a$ assumes both of the values $u$ and $v$, then it is ultimately periodic and hence bounded. If $A^{x} a$ is not bounded, then it must omit one of each pair of values $u, v$ for which $A u=A v$. Hence any function satisfying the condition (2) of the lemma must omit infinitely many values. Therefore every univalent function which is obtained from $A$ by substitution and recursion must omit infinitely many values. Thus, we cannot define $S$.

We have shown that there is no function $A$ from which we can obtain both $K$ and $S$ by substitution and recursion. Hence there is no function $A$ which can generate all primitive recursive functions of one variable.

Berkeley, Calif. 